\title{
LECTIN BINDING PATTERNS IN NORMAL, DYSPLASTIC AND HELICOBACTER PYLORI INFECTED GASTRIC MUCOSA
}

\author{
S. Vernygorodskyi*, V. Shkolnikov, D. Suhan \\ Department of Pathological Anatomy, Forensic Medicine and Law, National Pirogov Memorial Medical \\ University, Vinnytsia 21027, Ukraine
}

\begin{abstract}
Aim: To analyze the glycoprotein binding sites of the gastric mucosa and its secreted mucus using lectin histochemistry in patients with chronic non-atrophic gastritis (CNAG) associated or not-associated with Helicobacter pylori infection with or without dysplasia. Materials and Methods: In order to identify the areas with glycoconjugates expression in gastric mucosa, 6 lectins (Canavalia ensiformis agglutinin - Con A, Sambucus nigra agglutinin - SNA, wheat germ agglutinin - WGA, soybean agglutinin - SBA, Helix pomatia agglutinin - HPA, peanut agglutinin - PNA) were used. Carbohydrate determinants were visualized according to the lectin-peroxidase-diaminobenzidine staining protocol. Biopsy material was obtained and processed by conventional histological methods. The samples from 84 patients $(54$ with CNAG) with low $(n=34)$ and high grade $(n=20)$ dysplasia, 38 patients were $\mathrm{H}$. pylori-infected and 26 patients $-\mathrm{H}$. pylori-noninfected) were used. The comparison group included 30 persons with CNAG without dysplasia (16 patients $\mathrm{H}$. pylori-infected and 14 - noninfected). Results: In comparison to normal gastric mucosa, a low affinity of Con A was shown in $80 \%$ of patients with non-infected CNAG and $90 \%$ of $\mathrm{H}$. pylori associated CNAG. In 70\% of $\mathrm{H}$. pylori-infected patients with CNAG and low grade dysplasia there was an increase of SNA expression compared with noninfected patients $(p<0.05)$. Regarding SBA labeling no differences were detected in the studied groups $(p<0.05)$. In $H$. pylori infected patients with CNAG and low grade dysplasia, WGA, HPA and PNA showed a strong reactivity with the gastric mucosa cells in 80; 75\%, and $60 \%$ of patients, respectively. Conclusion: We suggest that a set of lectins in reaction with gastric epithelial and glandular cells can be used as a tool to obtain information about the dysplastic changes of the gastric mucosa and may offer new insight into gastric carcinogenesis and precancerous lesions treatment.
\end{abstract}

Key Words: lectins, Helicobacter pylori, gastric mucosa, dysplasia.

Helicobacter pylori (H. pylori) infection is highly prevalent in many developing countries, including those of Eastern Europe. In 1994, based on epidemiologic evidence, the International Agency for Research on Cancer classified $H$. pylori as a class I carcinogenic agent. The increasing antibiotic resistance associated with $H$. pylori eradication by antimicrobial therapy necessitates the search for the alternative strategies such as those based on blocking bacterial adhesion to host receptors. Knowing that infection and persistent colonization of human gastric mucosa by $\mathrm{H}$. pylori is highly dependent on bacterial adhesion to gastric epithelial cells, an effort should be made in order to better understand which bacterial lectin-host glycan interactions are truly critical for adhesion. Mucin type-O glycans have been demonstrated to be crucial players in the interaction between the bacteria and host gastric epithelial cells [1]. A strategy of carbohydrate-dependent inhibition of $H$. pylori colonization using porcine milk has been tested with success in mice, suggesting that milk from certain pig breeds may have a therapeutic

\section{Submitted: February 15, 2017. \\ *Correspondence: E-mail: medservervit@gmail.com Tel.: 0679565453}

Abbreviations used: CC - chief cells; CNAG - chronic nonatrophic gastritis; Con A - Canavalia ensiformis agglutinin; Gal - galactose; GalNac - N-acetyl-galactosamine; GlcNac - N-acetyl-glucosamine; HPA - Helix pomatia agglutinin; H. pylori - Helicobacter pylori; Man - mannose; MNC - mucous neck cells; NeuNAc - N-acetylneuraminic acid; PBS - phosphate buffered saline; PE - parietal exocrinocytes; PIE - pyloric exocrinocytes; PNA - peanut agglutinin; SBA - soybean agglutinin; SFE - superficial and foveolar cells; SNA - Sambucus nigra agglutinin; WGA - wheat germ agglutinin. and/or prophylactic effect on $H$. pylori infection. Lectin histochemistry is reported to be a sensitive method for studying the carbohydrate composition of glycoconjugates [2]. Lectins that identify and localize a variety of terminal carbohydrate structures on fixed sections of tissue are available [3]. Thus, it was hypothesized that this same technique would permit the identification of differentiation-associated structures in mucins of the stomach. Therefore, a study was designed in which mucosal biopsies were obtained from patients with chronic non-atrophic $H$. pylori-associated gastritis without dysplasia and compared with sections obtained from the dysplastic gastric mucosa.

\section{MATERIALS AND METHODS}

Patients and methods. Within one year 84 patients in endoscopy departments of Vinnytsia hospitals were examined to clarify the clinical diagnosis. There were 46 male (55\%) and 38 female (45\%) patients. Among these 84 patients we selected 54 with chronic non-atrophic gastritis (CNAG) with low $(n=34)$ and high grade $(n=20)$ dysplasia that were taken as a main group for further investigation. In this group 38 patients were diagnosed as $H$. pylori-infected and 26 patients as $H$. pylori-noninfected. The comparison group included 30 people with CNAG without dysplasia (16 patients were $H$. pylori-infected and $14-$ noninfected). The average age of patients examined was $41.4 \pm 3.1$ years. As the first-line therapy for eradication of $H$. pylori, all patients were treated by 10 -day triple therapy with omeprazole at a dose of $20 \mathrm{mg}$ (twice daily), amoxicillin at a dose of $1 \mathrm{~g} /$ day, and either 
clarithromycin or metronidazole at a dose of $500 \mathrm{mg}$ (twice daily).

All patients were thoroughly informed about the study that was approved by the local ethics committee. The informed consents were obtained from all research participants.

Multiple biopsies were performed during endoscopy (two biopsies from the body and antrum and one - from an area of the angle of the stomach) taking into account the requirements of the modified Sydney system of chronic gastritis diagnosis and stained areas of gastric mucosa with the following histological study of biopsies. Biopsy material was processed by conventional histological methods. To define dysplastic changes of gastric mucosa the following methods were used: staining with hematoxylin and eosin, combined alcian blue with $\mathrm{pH} 1.0$ and 2.5 together with the PAS reaction. $H$. pylori persistence was assayed with a rapid urease test and with a Pappenheim stain as well as with Romanovsky - Giemsa and toluidine blue staining.

Lectin histochemistry. The lectin panel included 6 conventional lectins (Table 1). All lectins and their peroxidase conjugates were prepared by Dr. V. Antonyuk (Lectinotest, Lviv, Ukraine) [4]. Carbohydrate determinants were visualized according to the lectinperoxidase-diaminobenzidine staining protocol [5].

Table 1. Lectins types and their carbohydrate specificity

\begin{tabular}{|c|c|c|c|}
\hline$\overline{\mathrm{N}}$ & $\begin{array}{l}\text { Lectin designation, } \\
\text { abbreviation }\end{array}$ & $\begin{array}{l}\text { Specific monosac- } \\
\text { charide }\end{array}$ & $\begin{array}{c}\text { Complementary oligo- } \\
\text { saccharide/polysac- } \\
\text { charide }\end{array}$ \\
\hline$\overline{1}$ & $\begin{array}{l}\text { Canavalia ensiformis } \\
\text { agglutinin, Con A }\end{array}$ & aDMan/ßDGIC & Glycogen \\
\hline 2 & $\begin{array}{l}\text { Sambucus nigra ag- } \\
\text { glutinin, SNA }\end{array}$ & NeuNAc( $(\alpha 2-6)$ DGal & $\begin{array}{c}\operatorname{NeuNAc}(\alpha 2-6) \\
\operatorname{Gal}(\beta 1-4) \operatorname{GlcNAc}(\beta 1-2)\end{array}$ \\
\hline 3 & $\begin{array}{l}\text { Soybean agglutinin, } \\
\text { SBA }\end{array}$ & $a D G a I N A c>b D G a l N A c$ & $\begin{array}{c}\text { GalNAc }(\alpha 1-3) \\
\text { Gal }(\beta 1-3) \text { GalNAc }\end{array}$ \\
\hline 4 & $\begin{array}{l}\text { Wheat germ aggluti- } \\
\text { nin, WGA }\end{array}$ & DGIcNAc $>$ NeuNAc & $\begin{array}{c}\text { NeuNAc }(\alpha 2-6) \\
\operatorname{Gal}(\beta 1-4) \\
\text { GIcNAc,Man }(\beta 1-4) \\
\text { GIcNAc }(\beta 1-4) \text { GIcNAc } \\
12\end{array}$ \\
\hline 5 & $\begin{array}{l}\text { Helix pomatia aggluti- } \\
\text { nin, HPA }\end{array}$ & aDGaINAc & GalNAc $(\alpha 1-3)$ GalNAc \\
\hline 6 & $\begin{array}{l}\text { Peanut agglutinin, } \\
\text { PNA }\end{array}$ & DGal & DGal $(\beta 1-3)$ GalNAc \\
\hline
\end{tabular}

Note: Man - mannose; NeuNAc - N-acetylneuraminic acid; GalNac - Nacetyl-galactosamine; GlcNac - N-acetyl-glucosamine; Gal - galactose.

In detail, deparaffinized sections were incubated for 20 min in methanol containing $0.3 \% \mathrm{H}_{2} \mathrm{O}_{2}$ to block activity of endogenous peroxidase, transferred through graded ethanol brought to phosphate buffered saline

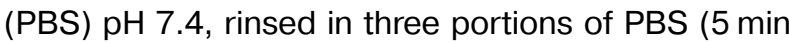
each), and incubated for $45 \mathrm{~min}$ with lectin-peroxidase conjugates (dilution $25-50 \mathrm{mg} / \mathrm{ml}$ in PBS) in a moist chamber at room temperature. Lectin receptor sites were visualized in PBS, containing $0.05 \%$ diaminobenzidine (Sigma, St. Louis, MO, USA) and $0.015 \% \mathrm{H}_{2} \mathrm{O}_{2}$. Then the slides were twice washed in distilled water, and after dehydration mounted in balsam. The specificity of histochemical reactions was controlled by: (1) omitting lectin-peroxidase from the staining protocol; and (2) pre-incubation of tissue sections prior to lectin labeling for 60 min in $1 \% \mathrm{HIO}_{4}$ (Reanal, Budapest, Hungary) for oxidative damage of carbohydrate determinants.
In both cases, staining results were negative. Microscopic investigation was performed using OLIMPUS BX 41 microscope (Japan). For the evaluation of lectin binding, two investigators performed the analysis independently, blinded to lectin type. Binding intensity was represented in the semiquantitative scale as follows: ++ strong, + positive, - negative labeling.

Statistical analysis. Results of lectinohistochemical alterations were compared to the clinicopathologic features using $\chi^{2}$ test with two tailed $p$ value, $p<0.05$ was considered as significant.

\section{RESULTS}

ConA demonstrated the most selective affinity for the superficial mucous cells, as well as for fundus and antrum in normal gastic mucosa with weak expression and loss of labeling in $80 \%$ of patients with non-infected CNAG and $90 \%$ of $H$. pylori associated CNAG. ConA, which binds specifically to Man and $\beta$ Glc-linked oligosaccharides, did not stain mucus-producing cells in the areas of dysplasia but stained the background weakly. The pyloric exocrinocytes (PIE) of glands were not stained with the ConA procedure (Table 2, 3).

Table 2. Lectin binding results of the fundus and antrum of the stomach in normal mucosa and non-atrophic gastritis

\begin{tabular}{|c|c|c|c|c|c|c|c|}
\hline \multirow{3}{*}{ Lectins } & \multicolumn{7}{|c|}{ Normal mucosa $H$. pylori-negative } \\
\hline & \multicolumn{4}{|c|}{ FUNDUS } & \multicolumn{3}{|c|}{ ANTRUM } \\
\hline & SFC & MNC & $\mathrm{CC}$ & $\mathrm{PE}$ & SFC & MNC & PIE \\
\hline ConA & ++ & + & $\overline{-}$ & - & + & $\overline{-}$ & - \\
\hline SNA & - & - & - & - & - & - & - \\
\hline SBA & + & + & - & + & + & + & + \\
\hline WGA & + & - & + & - & - & - & - \\
\hline HPA & + & - & - & - & - & + & - \\
\hline PNA & - & + & - & - & - & - & - \\
\hline
\end{tabular}

\begin{tabular}{lccccccc}
\hline & \multicolumn{9}{c}{ Gastritis $H$. pylori-negative } \\
\cline { 2 - 8 } Lectins & \multicolumn{9}{c}{ FUNDUS } & \multicolumn{5}{c}{ ANTRUM } \\
\cline { 2 - 8 } & SFC & MNC & CC & PE & SFC & MNC & PIE \\
\hline ConA & + & - & - & - & + & - & - \\
SNA & - & - & - & - & + & + & - \\
SBA & + & + & - & - & ++ & + & + \\
WGA & + & - & + & - & - & - & - \\
HPA & - & - & - & - & - & ++ & - \\
PNA & + & ++ & - & - & + & - & - \\
\hline
\end{tabular}

Note: SFE - superficial and foveolar cells; MNC - mucous neck cells; CC chief cells; PE - parietal exocrinocytes; PIE - pyloric exocrinocytes; - no expression, + weak expression, + + strong expression.

Table 3. Lectin binding results of the fundus and antrum of the stomach in non-atrophic $H$. pylori gastritis

\begin{tabular}{|c|c|c|c|c|c|c|c|}
\hline \multirow{4}{*}{ Lectins } & \multicolumn{7}{|c|}{ H. pylori-positive } \\
\hline & \multicolumn{7}{|c|}{ Low grade dysplasia } \\
\hline & \multicolumn{4}{|c|}{ FUNDUS } & \multicolumn{3}{|c|}{ ANTRUM } \\
\hline & SFC & MNC & $\mathrm{CC}$ & $\mathrm{PE}$ & SFC & MNC & PIE \\
\hline$\overline{\text { ConA }}$ & + & + & - & - & ++ & ++ & + \\
\hline SNA & - & - & - & - & + & + & - \\
\hline SBA & + & + & - & - & ++ & + & + \\
\hline WGA & + & - & + & - & + & + & - \\
\hline HPA & - & + & - & - & - & + & - \\
\hline PNA & - & + & - & - & + & - & - \\
\hline \multirow{4}{*}{ Lectins } & \multicolumn{7}{|c|}{ H. pylori-positive } \\
\hline & \multicolumn{7}{|c|}{ High grade dysplasia } \\
\hline & \multicolumn{4}{|c|}{ FUNDUS } & \multicolumn{3}{|c|}{ ANTRUM } \\
\hline & SFC & MNC & $\mathrm{CC}$ & $\mathrm{PE}$ & SFC & MNC & PIE \\
\hline ConA & - & + & - & - & + & ++ & + \\
\hline SNA & + & + & - & - & + & ++ & - \\
\hline SBA & + & + & - & - & ++ & + & + \\
\hline WGA & + & ++ & ++ & - & + & ++ & + \\
\hline HPA & ++ & + & ++ & - & ++ & ++ & + \\
\hline PNA & ++ & ++ & - & - & + & ++ & + \\
\hline
\end{tabular}

Note: SFE - superficial and foveolar cells; MNC - mucous neck cells; CC chief cells; PE - parietal exocrinocytes; PIE - pyloric exocrinocytes; - no expression, + weak expression, + + strong expression. 
SNA. There was no positive staining in antral and fundic mucins in normal gastric mucosa, however in the cytoplasm of the mucous neck cells (MNC) of the stomach antrum we revealed weak labeling in $40 \%$ patients with non-infected CNAG and high grade dysplasia and in $70 \%$ of $H$. pylori-infected CNAG and low grade dysplasia. SNA expression increased as compared with non-infected patients $(p<0.05)$.

SBA. MNC were intensely stained with SBA but in other cellular compartments of the gastric mucosa the SBA labeling was faint. Expression in the antrum was generally similar to that in the fundic gland mucosa, no differences $(p>0.05)$ were detected in the studied groups.

WGA. The presence of terminal GIcNAc was detected by WGA. Only chief cells (CC) and some superficial and foveolar cells (SFC) bound with WGA in normal gastric mucosa, non-infected CNAG and $H$. pylori infected CNAG with high grade dysplasia. In $80 \%$ of $H$. pylori infected patients with CNAG and low grade dysplasia the WGA showed a strong reactivity in the supranuclear portion and apical cytoplasm of MNC and CC of the gastric mucosa $(p<0.05)$.

HPA. In normal mucosa and non-infected CNAG the expression of HPA has not been defined in a majority of stomach cells but strong staining was detected at the supranuclear region of MNC while, the cytoplasm was slightly positive. In $75 \%$ of $H$. pylori-infected CNAG with low grade dysplasia the increased binding intensity in SFC, MNC and PIE was observed $(p<0.05)$.

PNA. Galactose residues were detected by PNA binding and only MNC were slightly positive in all investigated groups. The whole cytoplasm of the MNS appeared to react with PNA; the reaction was strong in the neck region, but the staining faded away towards the basal region. In $60 \%$ of patients with $\mathrm{H}$. pyloriinduced CNAG and low grade dysplasia PNA intensely labeled cytoplasm of SFC, MNS and PIE was revealed $(p<0.05)$.

\section{DISCUSSION}

The findings implicate that bacterial lectins sharing the same carbohydrate specificity can attach to the mucin oligosaccharides in normal and inflamed mucosa thus eliminating the need for the bacterium to switch lectin expression during the infection process. With the increasing prevalence of antibiotic-resistant organisms, new strategies to combat infection are being sought. One such strategy is the use of antiadhesive molecules targeting the primary step of infection, i.e. adhesion of the microorganism to the host cells due to binding of it with glycoprotein sites of the gastric mucosa. As bacterial adhesion is primarily mediated by the interaction of lectin-like molecules with highly specific carbohydrate structures present on gastric mucin and cell surfaces, carbohydrate-based compounds are ideal candidates for antiadhesives [6].

In our research the best selectivity of SFC labeling was obtained with ConA, but all infected and noninfected patients with CNAG demonstrated the ConA reduction of ConA binding. Based on SNA expression, sialated mucins were not present in normal gastric mucosa but our results are consistent with Macartney [7] studies demonstrating increased sialation of membrane glycoproteins following dysplasia.

In our opinion, one of the peculiarities of HPA lectin labeling is a strong binding to the supranuclear, Golgi-region in the undifferentiated or activated forms of the neck region cells, which is most common in the patients with $H$. pylori-associated CNAG and low grade dysplasia. The development of low grade dysplasia is not accompanied by important changes in glycosylation, however high grade dysplasia has its own characteristic patterns of glycosylation with loss of ConA expression, sialation of membrane glycoproteins and positive WGA, HPA and PNA labeling in dysplastic areas. This hypothesis is supported by the finding that exposure of HPA in undifferentiated cells of neck regions in $H$. pylori infected patients induces the formation of "immature" glycoproteins. The complex mechanisms underlying precancerous changes in the gastric mucosa, which are related to cancer development and a putative contribution of lectins is a field for further investigations.

\section{REFERENCES}

1. Magalhres A, Reis CA. Helicobacter pylori adhesion to gastric epithelial cells is mediated by glycan receptors. Braz J Med Biol Res 2010; 43: 611-8.

2. Sabine A, Herbert K, Klaus K, et al. Merging carbohydrate chemistry with lectin histochemistry to study inhibition of lectin binding by glycoclusters in the natural tissue context. Histochem Cell Biol 2016; 145: 185-99.

3. Shimamoto C, Weinstein WM, Boland CR. Glycoconjugate expression in normal, metaplastic, and neoplastic human upper gastrointestinal mucosa. J Clin Invest 1987; 80: 1670-8.

4. Antonyuk VO, Yastchenko AM, Antonyuk RV, et al. Carbohydrate specificity of lectin, purified from the fruiting bodies of Mycena pura /Fr./Kumm. and its use in histochemical investigation. Biopolym Cell 2009; 25: 466-75.

5. Brooks SA, Leathem AJC, Shumacher U. Lectin histochemistry: a concise practical handbook. Oxford: BIOS, 1997.

6. Mun Fai Loke, Sook Yin Lui, Bee Ling Ng, et al. Antiadhesive property of microalgal polysaccharide extract on the binding of Helicobacter pylori to gastric mucin. FEMS Immunol Med Microbiol 2007; 50: 231-8.

7. Macartney JC. Lectin histochemistry of galactose and $\mathrm{N}$-acetyl-galactosamine glycoconjugates in normal gastric mucosa and gastric cancer and the relationship with $\mathrm{ABO}$ and secretor status. J Pathol 1986; 150: 135-44. 